\title{
Brownian Motion \& the Stochastic Behavior of Stocks
}

\author{
Pantelis Tassopoulos ${ }^{1}$, Yorgos Protonotarios ${ }^{2}$ \\ ${ }^{1}$ Department of Mathematics, Imperial College London, London, UK \\ ${ }^{2}$ Department of Mathematics, University College London, London, UK \\ Email: pantelis.tassopoulos20@imperial.ac.uk, yorgos.protonotarios.20@ucl.ac.uk
}

How to cite this paper: Tassopoulos, P. and Protonotarios, Y. (2022) Brownian Motion $\&$ the Stochastic Behavior of Stocks. Journal of Mathematical Finance, 12, 138-149. https://doi.org/10.4236/jmf.2022.121009

Received: December 30, 2021

Accepted: February 12, 2022

Published: February 15, 2022

Copyright $\odot 2022$ by author(s) and Scientific Research Publishing Inc. This work is licensed under the Creative Commons Attribution International License (CC BY 4.0).

http://creativecommons.org/licenses/by/4.0/

\begin{abstract}
In this paper, we test the effectiveness of predicting the behavior of stocks utilizing stochastic calculus. We begin by exploring the intuition of Brownian motion by explaining its birth through the observations of Robert Brown and later through Bachelier's work on its applications to the financial market and finally its rigorous and concretized form proposed by Norbert Wiener. The aforementioned motivates a stochastic differential equation to model the future price fluctuations of a stock wherein Itô integration is prominent and consequently expanded upon. The final part of this paper focuses on the accuracy of the model by back testing it with Apple stock and deriving a correlation coefficient.
\end{abstract}

\section{Keywords}

Brownian Motion, Stochastic Calculus, Stochastic Differential Equation, Stocks, Itô Integral

\section{Introduction}

This paper begins with the contribution of the works of Robert Brown, Louis Bachelier and Norbert Wiener which is then followed by the mathematical definition of Brownian Motion and accompanied with the discretized form of a stochastic differential equation. Furthermore, Chapter 5 focuses on the introduction to certain preliminary concepts such as sigma algebras, filtrations and $L^{p}$ spaces. Finally, we construct the Itô integral and consequently apply it to the limiting form of the stochastic differential equation. Finally, Chapter 7 tests the equation against Apple Stock. 


\section{Literature Review}

\subsection{Robert Brown}

In 1827, a botanist by the name of Robert Brown was examining the motion of grains of pollen suspended under water from a species of plants. Curiously, Brown observed that the motion of particles ejected from these pollen grains was "jittery"; this was the first ever recorded case of such motion and was thus subsequently named "Brownian". The idea behind this type of motion being that the trajectory follows a completely random and "unpredictable" path. Ever since, the concept of an unpredictable and random trajectory has been utilized in numerous fields including financial mathematics in the modeling of stock behavior [1].

\subsection{Louis Bachelier}

Several decades later, in 1900, Louis Bachelier built the foundation of mathematical finance by integrating Brownian motion with the fluctuation in the price of a stock. He postulated that two ideas should be considered when exploring the future value of an asset. First, how a collection of anterior (past) events influences the asset and second, how the probability of unknown future events could affect it [2]. For example, the C.E.O of Apple just got replaced yesterday; that is an anterior event that could influence the future price of an Apple stock. On the other hand, if a power outage occurs tomorrow in one of Apple's factories and halts production, that is an unknown future event that might affect the price of the stock and falls into the second category. It is said that the fluctuation in the price of a stock attributed to the latter follows Brownian motion as it is seemingly unpredictable and random. Bachelier only focused on the second idea since the anterior events were not meaningful because Bachelier defined the mathematical expectation for an asset to rise or fall to be zero since the market constitutes of a pool of people that trade with opposite beliefs on the future value of an asset [2]. For example, suppose a person is buying a call option for an asset, they hence believe that its price will rise. The person on the other side of the option believes it will fall otherwise they would not be selling the option. Hence, since they are both aware of past events that can influence the asset and have contradictory beliefs on how it will perform, meaning the mathematical expectation of the change in value of the underlying asset is neither positive nor negative. The market is therefore said to be fair [2].

\subsection{Norbert Wiener}

The above considerations by Bachelier were expanded upon within the framework of physics by American mathematician Norbert Wiener in his seminal work on "differential spaces" [3]. This was justified by arguments pertaining to the motion of a particle suspended in a fluid, where the movement of said particle depended on impulses by fluid particulates-akin to Brown's scenario and the initial velocity of the particle, although the influence of the latter was deemed negligible by Einstein. For simplicity, one can assume that the par- 
ticle is constrained in one dimension and so are the impulses. Due to the nature of the situation, Wiener, following Einstein assumed that the displacement of the particle between any two-time instants had no bias in any direction and large movements relative to the time scale were unlikely. This prompted the use of the normal distribution as a way of describing the above behavior. The use of the normal distribution and the "independence" of the displacement of the particle in disjoint time intervals are crucial in the rigorous definition of Brownian motion given below.

\section{Brownian Motion}

In probability theory, one usually considers three objects when setting up a probability space; namely, the sample space $\Omega$, that is the collection of outcomes of a random process, a sigma algebra $\mathcal{F}$, a set containing all measurable events and a probability measure $\mathbb{P}$ that measures the probability of said events that obeys certain axioms as laid out by Kolmogorov [4]. The above are combined to form the Probability Space $(\Omega, \mathcal{F}, \mathbb{P})$.

A stochastic process defined on a probability space $(\Omega, \mathcal{F}, \mathbb{P})$ is a measurable function $X:[0, \infty) \times \Omega \rightarrow \mathbb{R}$. More specifically, a stochastic process $\mathfrak{B}(t, \omega)$ is called a Brownian motion if it satisfies the following conditions [5]:

1) $\mathbb{P}(\omega ; \mathfrak{B}(0, t)=0)=1$

2) For any $0 \leq s<t$, the random variable $\mathfrak{B}(t)-\mathfrak{B}(s)$ is normally distributed with mean 0 and variance $t-s$, i.e.,

$$
\mathbb{P}(a \leq \mathfrak{B}(t)-\mathfrak{B}(s) \leq b)=\frac{1}{\sqrt{2 \pi(t-s)}} \int_{a}^{b} \mathrm{e}^{\frac{-x^{2}}{2(t-s)}} \mathrm{d} x
$$

3) $\mathfrak{B}(t, \omega)$ has independent increments, i.e. for any $0 \leq t_{1} \leq t_{2} \leq \cdots \leq t_{n}$, the random variables

$$
\mathfrak{B}\left(t_{1}\right), \mathfrak{B}\left(t_{2}\right)-\mathfrak{B}\left(t_{1}\right), \cdots, \mathfrak{B}\left(t_{n}\right)-\mathfrak{B}\left(t_{n-1}\right)
$$

are independent and identically distributed, as per II.

4) Almost all sample paths of $\mathfrak{B}(t, \omega)$ are continuous functions, i.e.:

$$
\mathbb{P}(\{\omega \in \Omega \mid \mathfrak{B}(., \omega) \text { is continuous }\})=1
$$

Note that the dependence on $\omega$ was dropped in property II and III for simplicity reasons.

\section{Applications to the Stock Market}

We now consider a stock that is tradable on a stock market, such as an S\&P 500 stock like Apple and model it by a stochastic process $\mathcal{Y}_{t}(\omega):[0, \infty) \times \Omega \rightarrow \mathbb{R}$ on the probability space $(\Omega, \mathcal{F}, \mathbb{P})$. One is prompted to ask

What factors drive the process $\mathcal{Y}_{t}$ ?

Well, an attempt at answering the above is to view the stock price $\mathcal{Y}_{t}$ as a 
sum of a deterministic component and a stochastic component containing "noise" meant to represent an underlying uncertainty. Thus, for a small increment in time from $t$ to $t+\mathrm{d} t$, the change in $\log \mathcal{Y}_{t}$ i.e., $d \log \mathcal{Y}_{t}$ is given by

$$
\mathrm{d} \log \mathcal{Y}_{t}=\gamma(t) \cdot \mathrm{d} t+\sigma\left(t, \mathcal{Y}_{t}\right) \cdot \text { "noise" }
$$

where $\gamma(t)$ is the growth rate of a stock which is deterministic and $\sigma\left(t, \mathcal{Y}_{t}\right)$. "noise" is the volatility of the stock and is the stochastic component [6]. The noise part can be modeled by a Brownian Motion $\mathfrak{B}_{t}$, following Bachelier and Wiener. Thus, the above equation can be recast in the following form [7]

$$
\mathrm{d} \log \mathcal{Y}_{t}=\gamma(t) \cdot \mathrm{d} t+\sigma\left(t, \mathcal{Y}_{t}\right) \cdot \mathrm{d} \mathfrak{B}(t)
$$

where $\mathrm{d} \mathfrak{B}_{t}=\mathfrak{B}_{t+\mathrm{d} t}-\mathfrak{B}_{t}$. Equation 1 is an example of a stochastic differential equation [6]. In order to make precise what we mean by this, we consider a discretisation of the problem and consider the interval $[0, t]$ where $t \geq 0$. We further impose a partition $t_{0}=0<t_{1}<t_{2}<\cdots<t_{k-1}<t=t_{n}$ of the interval and equation 1 to mean:

$$
\log \mathcal{Y}_{t_{k}}-\log \mathcal{Y}_{t_{k-1}}=\gamma\left(t_{k-1}\right) \cdot\left(t_{k}-t_{k-1}\right)+\sigma\left(t_{k-1}, \mathcal{Y}_{t_{k-1}}\right) \cdot\left(\mathfrak{B}_{t_{k}}-\mathfrak{B}_{t_{k-1}}\right)
$$

for $k$ ranging from 0 to $n-1$. A summation of the index $k$ yields the process

$$
\begin{aligned}
I_{n}\left[\mathcal{Y}_{t}\right](.) & =\log \mathcal{Y}_{t_{n} \equiv t}-\log \mathcal{Y}_{t_{0}} \\
& =\sum_{k=0}^{n-1} \gamma\left(t_{k-1}\right) \cdot\left(t_{k}-t_{k-1}\right)+\sum_{k=0}^{n-1} \sigma\left(t_{k-1}, \mathcal{Y}_{t_{k-1}}\right) \cdot\left(\mathfrak{B}_{t_{k}}-\mathfrak{B}_{t_{k-1}}\right)^{[6]}
\end{aligned}
$$

Now, in a certain sense, we have "integrated the process" and have obtained an expression for the process at some time $t$, given an initial time $t_{0}$. Indeed, two summations that appear are reminiscent of discrete approximations to Riemann-Stieltjes integrals. One is tempted to take a limit of such partitions $\pi_{n}$ with mesh $\left|\pi_{n}\right| \equiv \max \left(t_{i}-t_{i-1}\right) \rightarrow 0$ and obtain the corresponding equation

$$
\log \mathcal{Y}_{t_{n} \equiv t}-\log \mathcal{Y}_{t_{0}}=\int_{0}^{t} \gamma(t) \mathrm{d} t+\int_{0}^{t} \sigma\left(t, \mathcal{Y}_{t}\right) \mathrm{d} \mathfrak{B}_{t}
$$

The aim of the next chapter is to show that for suitably well behaved $\gamma(t)(\omega)$ and $f(t, \omega) \equiv \sigma\left(t, \mathcal{Y}_{t}(\omega)\right)$, such a limit exists in $L^{2}(\Omega)$, the space of all square integrable random variables on $\Omega$. Making this intuition precise leads to the Itô Integral, which will be discussed below.

\section{Preliminaries}

\section{1. $\sigma$-Algebras}

Consider the space $X$. A $\sigma$-algebra $\mathcal{A}$ on $X$ [8] is a set of subsets of $X$ satisfying the following conditions:

1) $X, \varnothing \in \mathcal{A}$;

2) if $A \in \mathcal{A}$ then $\bar{A} \in \mathcal{A}$;

3) if $A_{j} \in \mathcal{A}$ for $j \in \mathbb{N}$ then $\bigcup_{j \in \mathbb{N}} A_{j} \in \mathcal{A}$.

A generator $\mathcal{G}$ is a set of arbitrary subsets such that these subsets generate a $\sigma$-algebra denoted by $\sigma(\mathcal{G})$ [8]. This $\sigma$-algebra is also known as the smallest or 
minimal $\sigma$-algebra. It is defined as:

$$
\sigma(\mathcal{G}):=\bigcap_{\substack{\mathcal{H}_{k} \text { is a } \sigma \text {-alg } \\ \mathcal{G} \subset \mathcal{H}_{k}}} \mathcal{H}_{k}
$$

for an arbitrary index $k$, that is the intersection of all sigma algebras that contain $\mathcal{G}$.

\subsection{Filtrations \& $\mathbb{F}$-Adapted Processes}

Consider the family $\mathbb{F}:=\left\{\mathcal{F}_{t}\right\}_{t \in T}$ of $\sigma$-(sub)algebras of $\mathcal{F}$ defined on a measurable space $(\Omega, \mathcal{F})$ is called a filtration if for all $s, t \in T$ such that $s \leq t$,

$$
\mathcal{F}_{s} \subseteq \mathcal{F}_{t}
$$

where $T$ is an index set in any of the following: $\mathbb{R}, \mathbb{R}_{+}, \mathbb{N}, \mathbb{Z}$. [8]

An $\mathcal{F}_{t}$-measurable function $Z$ satisfies:

$$
Z^{-1}(\mathcal{B}(\mathbb{R})) \subset \mathcal{F}_{t}
$$

which is a shorthand notation for:

$$
Z^{-1}(B) \in \mathcal{F}_{t}, \forall B \in \mathcal{B}(\mathbb{R})
$$

where $\mathcal{B}(\mathbb{R})$ is the standard Borel $\sigma$-algebra. Define a function $h:[0, \infty) \times \Omega \rightarrow \mathbb{R}$ with a filtration $\mathbb{F} \subset \Omega$. The function is then called $\mathcal{F}_{t}$-adapted if it is $\mathcal{F}_{t}$-measurable $\forall t \geq 0 \quad$ [8].

\subsection{A.M - G.M Inequality \& Lebesgue Integral}

The A.M - G.M inequality states the following:

$$
\frac{a^{\prime}+b^{\prime}}{2} \geq \sqrt{a^{\prime} b^{\prime}}
$$

for $a^{\prime}, b^{\prime} \in \mathbb{R}^{+}$. Now replace $a^{\prime}=a^{2}$ and $b^{\prime}=b^{2}$. The inequality then becomes:

$$
\frac{a^{2}+b^{2}}{2} \geq|a b|
$$

for $a, b \in \mathbb{R}$. This can be extended to $\mathbb{P}$-measurable functions $f$ and $g$ in the context of integration. Using the fact that the Lebesgue integral with respect to the probability measure $\mathbb{P}$ is monotonic, we can apply it to the inequality yielding:

$$
\begin{aligned}
& \int_{\Omega}\left(f^{2}+g^{2}\right) \mathrm{d} \mathbb{P} \geq 2 \int_{\Omega}|f g| \mathrm{d} \mathbb{P} \\
& \Rightarrow \mathbb{E}\left[f^{2}+g^{2}\right] \geq 2 \mathbb{E}[|f g|]
\end{aligned} .
$$

\subsection{Lp Spaces}

Suppose $X: \Omega \rightarrow \mathbb{R}^{n}$ is a random variable and $p \in[1, \infty)$. The $L^{p}$ norm of $X$, $\|X\|_{L^{p}}$ is defined as [6]:

$$
\|X\|_{L^{p}}=\left(\int_{\Omega}|X(\omega)|^{p} \mathrm{~d} \mathbb{P}(\omega)\right)^{1 / p}=\left(\mathbb{E}\left(|X|^{p}\right)\right)^{1 / p} .
$$




\section{Itô Integration}

A process $\phi:[0, \infty) \times \Omega \rightarrow \mathbb{R}$ is called elementaryif:

$$
\phi(t, \omega)=\sum_{j=1}^{K-1} Z_{j}(\omega) \mathbb{1}_{\left(t_{i}, t_{i+1}\right]}(t)
$$

where $0 \leq t_{1}<t_{2}<\cdots<t_{K}<\infty$ and where $Z_{j}(1 \leq i \leq K)$ is a complex squareintegrable $\mathcal{F}_{t_{i}}$-measurable random variable. [8] The Itô integral for this process on the interval $[S, T]$ with $S, T \in[0, \infty)$ is defined as the following random variable:

$$
\int_{S}^{T} \phi(t, \omega) \mathrm{d} \mathfrak{B}_{t}=\sum_{j=1}^{K-1} Z_{j}(\omega)\left[\mathfrak{B}_{t_{j+1}}-\mathfrak{B}_{t_{j}}\right](\omega) .
$$

In our case, a square integrable random process is defined as

$$
\mathbb{E}\left[\int_{S}^{T}|\phi(t)|^{2} \mathrm{~d} t\right]<\infty .
$$

Define $\mathfrak{L}(S, T)$ to be the class of functions [6]:

$$
f:[0, \infty) \times \Omega \rightarrow \mathbb{R}
$$

such that:

1) $f(t, \omega)$ is $\mathcal{B}\left(\mathbb{R}^{+}\right) \times \mathcal{F}$-measurable;

2) $f(t, \omega)$ is $\mathcal{F}_{t}$-adapted;

3) $\mathbb{E}\left[\int_{S}^{T}|f(t, \omega)|^{2} \mathrm{~d} t\right]<\infty$.

\section{Construction of the Itô Integral}

We will know construct the Itô integral. We will omit the proofs of the steps needed for such a construction for the purpose of brevity. In brief, the construction comprises of approximation lemmas for processes $g \in \mathfrak{L}(S, T)$ in terms of elementary functions, and then one utilises the isometry property of the Itô integral for elementary processes and the completeness of the metric space $L^{2}(\Omega)$ to define a limit and call it the Itô integral.

\section{STEP I}

Let $g \in \mathfrak{L}(S, T)$ be bounded and continuous for all $\omega \in \Omega$. Then there exists elementary processes $\phi_{n} \in \mathfrak{L}(S, T)$ such that [6]

$$
\mathbb{E}\left[\int_{S}^{T}\left(g-\phi_{n}\right)^{2} \mathrm{~d} t\right] \rightarrow 0, \text { as } n \rightarrow \infty .
$$

\section{STEP II}

Let $h \in \mathfrak{L}(S, T)$ be bounded. Then there exist bounded functions $g_{n} \in \mathfrak{L}(S, T)$ such that they are continuous for all $\omega$ and $n$ and [6]

$$
\mathbb{E}\left[\int_{S}^{T}\left(h-g_{n}\right)^{2} \mathrm{~d} t\right] \rightarrow 0, \text { as } n \rightarrow \infty .
$$

\section{STEP III}

Let $f \in \mathfrak{L}(S, T)$. Then there exists a sequence $\left\{h_{n}\right\} \subset \mathfrak{L}(S, T)$ such that $h_{n}$ is bounded for each $n$ and [6] 


$$
\mathbb{E}\left[\int_{S}^{T}\left(f-h_{n}\right)^{2} \mathrm{~d} t\right] \rightarrow 0, \text { as } n \rightarrow \infty .
$$

Now, using the above steps we show that for $f, \phi_{n} \in \mathfrak{L}(S, T)$ :

$$
\mathbb{E}\left[\int_{S}^{T}\left|f-\phi_{n}\right|^{2} \mathrm{~d} t\right] \rightarrow 0, \text { as } n \rightarrow \infty .
$$

First, maintain the definition for $f, \phi_{n}, g$ and $h$ as they were in steps I, II and III. We have by section 4.3:

$$
\begin{aligned}
& \mathbb{E}\left[\int_{S}^{T}\left|f-\phi_{n}\right|^{2} \mathrm{~d} t\right] \\
& =\mathbb{E}\left[\int_{S}^{T}\left|\left(f-h_{k}\right)+\left(h_{k}-g_{m}\right)+\left(g_{m}-\phi_{n}\right)\right|^{2} \mathrm{~d} t\right] \\
& \leq 2 \mathbb{E}\left[\int_{S}^{T}\left|f-h_{k}\right|^{2} \mathrm{~d} t\right]+4 \mathbb{E}\left[\int_{S}^{T}\left|h_{k}-g_{m}\right|^{2} \mathrm{~d} t\right]+4 \mathbb{E}\left[\int_{S}^{T}\left|g_{m}-\phi_{n}\right|^{2} \mathrm{~d} t\right]
\end{aligned}
$$

where for now $h_{k}$ and $g_{m}$ are arbitrary functions. Let $\epsilon>0$ be arbitrary. By Step III, fix a $k$ large enough such that:

$$
\mathbb{E}\left[\int_{S}^{T}\left|f-h_{k}\right|^{2} \mathrm{~d} t\right]<\frac{\epsilon}{6}
$$

By Step II, fix an $m$ large enough such that:

$$
\mathbb{E}\left[\int_{S}^{T}\left|h_{k}-g_{m}\right|^{2} \mathrm{~d} t\right]<\frac{\epsilon}{12} .
$$

By Step I, fix an Nlarge enough such that for all $n \geq N$ :

$$
\mathbb{E}\left[\int_{S}^{T}\left|g_{m}-\phi_{n}\right|^{2} \mathrm{~d} t\right]<\frac{\epsilon}{12} .
$$

Hence, for all $n \geq N$, combining the above parts yields that

$$
\mathbb{E}\left[\int_{S}^{T}\left|f-\phi_{n}\right|^{2} \mathrm{~d} t\right]<\epsilon .
$$

We now will state without proof a key property of the Itô integral for elementary processes $\phi \in \mathfrak{L}(S, T)$, known as Itô Isometry and states the following and can be found in [6]

$$
\mathbb{E}\left[\left(\int_{S}^{T} \phi(t) \mathrm{d} \mathfrak{B}_{t}\right)^{2}\right]=\mathbb{E}\left[\int_{S}^{T} \phi(t)^{2} \mathrm{~d} t\right] .
$$

Now, armed with the above results, we are going to show that the sequence

$$
\left\{\int_{S}^{T} \phi_{n}(t, \omega) \mathrm{d} \mathfrak{B}_{t}\right\}_{n \in \mathbb{N}}
$$

is Cauchy in $L^{2}(\Omega)$, where the $\phi_{n}(t, \omega)$ are elementary approximants of $f(t, \omega)$ in the sense that $\mathbb{E}\left[\int_{S}^{T}\left(f-\phi_{n}\right)^{2} \mathrm{~d} t\right] \rightarrow 0$ as $n \rightarrow \infty$. Indeed,

$$
\begin{aligned}
& \left\|\int_{S}^{T}\left(\phi_{n}(t, \omega)-\phi_{m}(t, \omega)\right) \mathrm{d} \mathfrak{B}_{t}\right\|_{L^{2}(\Omega)}^{2} \\
& =\mathbb{E}\left[\left(\int_{S}^{T}\left(\phi_{n}(t, \omega)-\phi_{m}(t, \omega)\right) \mathrm{d} \mathfrak{B}_{t}\right)^{2}\right]=\mathbb{E}\left[\int_{S}^{T}\left(\phi_{n}(t, \omega)-\phi_{m}(t, \omega)\right)^{2} \mathrm{~d} t\right] \\
& \leq 2 \mathbb{E}\left[\int_{S}^{T}\left(f(t, \omega)-\phi_{n}(t, \omega)\right)^{2} \mathrm{~d} t\right]+2 \mathbb{E}\left[\int_{S}^{T}\left(f(t, \omega)-\phi_{m}(t, \omega)\right)^{2} \mathrm{~d} t\right] \rightarrow 0
\end{aligned}
$$


as $n, m \rightarrow \infty$ by the A.M-G.M. inequality, the approximation lemma and the isometry property of the Itô integral. The completeness of $L^{2}(\Omega)$ implies that there is a random variable $I[f]_{S}^{T} \in L^{2}(\Omega)$ such that

$$
\mathbb{E}\left[\left(I[f]_{S}^{T}-\int_{S}^{T} \phi_{n}(t, \omega) \mathrm{d} \mathfrak{B}_{t}\right)^{2}\right] \rightarrow 0
$$

as $n \rightarrow \infty$. We can now define the Itô integral of $f(t, \omega)$ as

$$
\int_{S}^{T} f(t, \omega) \mathrm{d} \mathfrak{B}_{t}:=I[f]_{S}^{T} \stackrel{L^{2}(\Omega)}{=} \lim _{n \rightarrow \infty} \int_{S}^{T} \phi_{n}(t, \omega) \mathrm{d} \mathfrak{B}_{t} .
$$

It is also important to mention that the above limit does not depend on the choice of approximants. So suppose the approximants $\phi_{n}(t, \omega)$ and $\psi_{n}(t, \omega)$ converge in the $L^{2}(\Omega)$ sense to $I_{1}[f]_{S}^{T}$ and $I_{2}[f]_{S}^{T}$ respectively, then we have

$$
\begin{aligned}
& \left\|I_{1}[f]_{S}^{T}-I_{2}[f]_{S}^{T}\right\|_{L^{2}(\Omega)}^{2} \\
& \leq\left\|\left(I_{1}[f]_{S}^{T}-\int_{S}^{T} \phi_{n}(t, \omega) \mathrm{d} \mathfrak{B}_{t}\right)-\left(I_{2}[f]_{S}^{T}-\int_{S}^{T} \phi_{n}(t, \omega) \mathrm{d} \mathfrak{B}_{t}\right)\right\|_{L^{2}(\Omega)}^{2} \\
& \leq\left\|I_{1}[f]_{S}^{T}-\int_{S}^{T} \phi_{n}(t, \omega) \mathrm{d} \mathfrak{B}_{t}\right\|_{L^{2}(\Omega)}^{2}+\left\|I_{2}[f]_{S}^{T}-\int_{S}^{T} \phi_{n}(t, \omega) \mathrm{d} \mathfrak{B}_{t}\right\|_{L^{2}(\Omega)}^{2} \\
& \leq\left\|I_{1}[f]_{S}^{T}-\int_{S}^{T} \phi_{n}(t, \omega) \mathrm{d} \mathfrak{B}_{t}\right\|_{L^{2}(\Omega)}^{2}+\left\|I_{2}[f]_{S}^{T}-\int_{S}^{T} \psi_{n}(t, \omega) \mathrm{d} \mathfrak{B}_{t}\right\|_{L^{2}(\Omega)}^{2} \\
& +\left\|\int_{S}^{T} \phi_{n}(t, \omega) \mathrm{d} \mathfrak{B}_{t}-\int_{S}^{T} \psi_{n}(t, \omega) \mathrm{d} \mathfrak{B}_{t}\right\|_{L^{2}(\Omega)}^{2} \rightarrow 0
\end{aligned}
$$

as $n \rightarrow \infty$. This is because

$$
\begin{aligned}
& \| \int_{S}^{T} \phi_{n}(t, \omega) \mathrm{d} \mathfrak{B}_{t}-\left.\int_{S}^{T} \psi_{n}(t, \omega) \mathrm{d} \mathfrak{B}_{t}\right|_{L^{2}(\Omega)} ^{2}=\mathbb{E}\left[\int_{S}^{T}\left(\phi_{n}-\psi_{n}\right)^{2}(t, \omega) \mathrm{d} t\right] \\
& \leq 2 \mathbb{E}\left[\int_{S}^{T}\left(\phi_{n}-f\right)^{2}(t, \omega) \mathrm{d} t\right]+2 \mathbb{E}\left[\int_{S}^{T}\left(\psi_{n}-f\right)^{2}(t, \omega) \mathrm{d} t\right] \rightarrow 0
\end{aligned}
$$

by the fact that $\phi_{n}$ and $\psi_{n}$ are approximants to $f$ and the definition of the Itô integral. This shows that $\left\|I_{1}[f]_{S}^{T}-I_{2}[f]_{S}^{T}\right\|_{L^{2}(\Omega)}^{2}=0$ which means that $\mathbb{P}\left(\left\{\omega \in \Omega \mid I_{1}[f]_{S}^{T}(\omega)=I_{2}[f]_{S}^{T}(\omega)\right\}\right)=1$, that is that the limits are "almost surely" equal, hence they are identified in $L^{2}(\Omega)$.

\section{Numerical Results}

Now, for some numerical results to buttress the theory, we consider the case where the stock price process $\mathcal{Y}_{t}$ from some initial time $t=0$ to some final time $t=T$ follows the law

$$
\log \mathcal{Y}_{t}-\log \mathcal{Y}_{0}=\int_{0}^{t} \Gamma \mathrm{d} t+\int_{0}^{t} \Sigma \mathrm{d} \mathfrak{B}_{t}
$$

for $t \in[0, T]$, where $\gamma(t)=\Gamma$ and $\sigma\left(\mathcal{Y}_{t}, t\right)=\Sigma$ are constants. The above formula is well defined as constant functions are members of the space $\mathfrak{L}(0, T)$ 
for which the Itô integral is defined. The above can be solved analytically, but a numerical treatment will be explored within the context of Apple's stock price.

In the following example, the model will be tested against historical data from January 12020 to December 312020 . $\Gamma$ and $\Sigma$ will be estimated using historical daily log-returns from 11 October 2007. By daily log-returns at a given date, we mean the natural logarithm of the ratio of the price at said day by the price at the previous date. First, we partition the period from 1 January 2020 to 31 December 2020 into

$$
\mathfrak{T}=\left\{T_{i}\right\}_{i=i^{\text {th }}} \text { trading day in the year }
$$

starting from $i=0$ and we call the collection of log-returns

$$
\log \mathfrak{R}=\left\{\log R_{t_{i}}\right\}_{t_{i} \in \mathfrak{T} T_{0}}
$$

where

$$
R=\frac{X_{i}}{X_{i-1}}
$$

and $X_{i}$ is the price of Apple's stock at some $t_{i} \in \mathfrak{T}\left\{T_{0}\right\}$. We use the above to compute $\Gamma$ and $\Sigma$ :

$$
\begin{gathered}
\Gamma=\overline{\log \mathfrak{R}}-\frac{\operatorname{Var}(\log \mathfrak{R})^{2}}{2} \\
\Sigma=\operatorname{Var}(\log \mathfrak{R})
\end{gathered}
$$

where $\overline{\log \mathfrak{R}}$ is the (sample) mean and $\operatorname{Var}(\log \mathfrak{R})$ the (sample) variance of log $\Re$ respectively. We are now able to consider discrete approximation to equation 1 . As per section 4 , we consider the discrete stock price process $\left.\mathcal{Y}_{t}\right|_{\mathfrak{T}}: \mathfrak{T} \times \Omega \rightarrow \mathbb{R}$ given by:

$$
\begin{aligned}
\left.\mathcal{Y}_{t_{n}}\right|_{\mathfrak{T}}(\omega)= & H_{t_{0}} \times \exp \left(\sum_{k=0}^{n-1} \gamma\left(t_{k-1}\right)(\omega) \cdot\left(t_{k}-t_{k-1}\right)\right) \\
& \times \exp \left(\sum_{k=0}^{n-1} \sigma\left(t_{k-1}, \mathcal{Y}_{t_{k-1}}(\omega)\right) \cdot\left(\mathfrak{B}_{t_{k}}(\omega)-\mathfrak{B}_{t_{k-1}}(\omega)\right)\right)
\end{aligned}
$$

where $\left.\mathcal{Y}_{t_{0}}\right|_{\mathfrak{T}}=H_{t_{0}}, t_{n} \in \mathfrak{T}$ and $\omega \in \Omega$. (Figure 1)

It is clear from Figure 2, the simulated paths capture most of the historical time series of Apple's stock price which is another indicator that the model is qualitatively speaking, a good approximation. However, only ten projections were performed, justifiably casting doubt on the statistical significance of the above result.

To try and quantify the above intuition, we will try and compute numerically the expected value, a central tendency indicator, $\mathbb{E}\left[\operatorname{Cor}\left(\left.\mathcal{Y}_{t}\right|_{\mathfrak{T}}, H_{t}\right)\right]$ of the correlation between $\left.\mathcal{Y}_{t}\right|_{\mathfrak{T}}$ and $\left\{H_{t}\right\}_{t \in \mathfrak{T}}-H_{t}$ in short the historical price time series of Apple Stock in the above period.

To achieve this, we consider $N$ independent and identically distributed copies $\left.\mathcal{Y}_{t}^{n}\right|_{\mathcal{T}}$ where $n \in[1, N] \cap \mathbb{N}$, of the discrete process $\left.\mathcal{Y}_{t}\right|_{\mathfrak{T}}$; intuitively, they correspond to $N$ distinct projections. The correlation between the historical data 
and the $n^{\text {th }}$ projection is denoted by

$$
\operatorname{Cor}\left(\left.\mathcal{Y}_{t}^{n}\right|_{\mathfrak{T}}, H_{t}\right)
$$

with cumulative mean

$$
\mathfrak{C o r}^{N}=\frac{\sum_{n=1}^{N} \operatorname{Cor}\left(\mathcal{Y}_{t}^{n}, H_{t}\right)}{N}
$$

taking $N \rightarrow \infty$, we obtain Figure 3 .

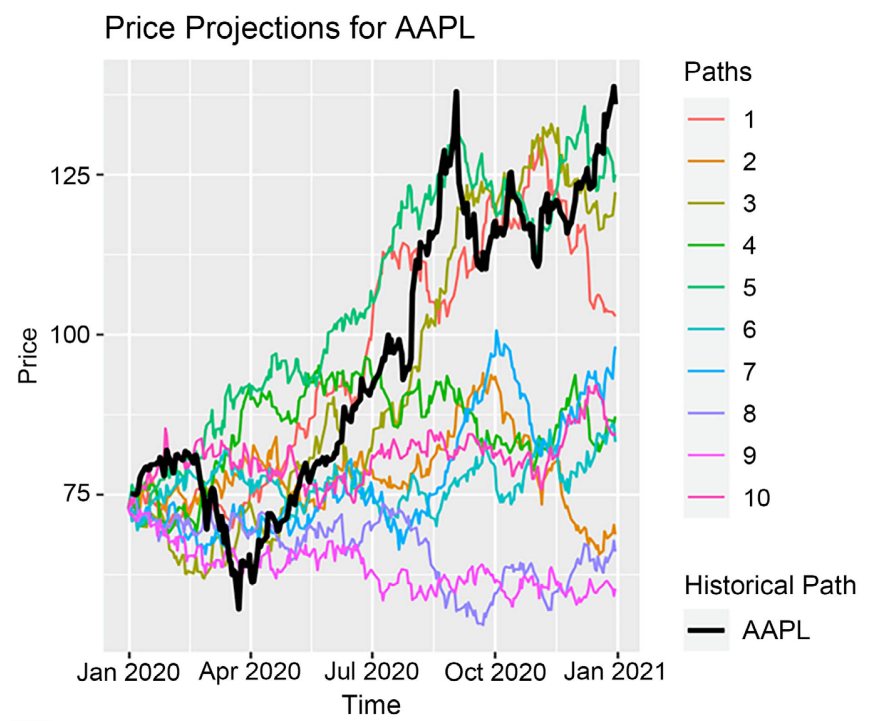

Figure 1. Plot Apple Stock Price (black) from January 2020 to December 312020 and projections (coloured) for 10 sample paths generated from the model.

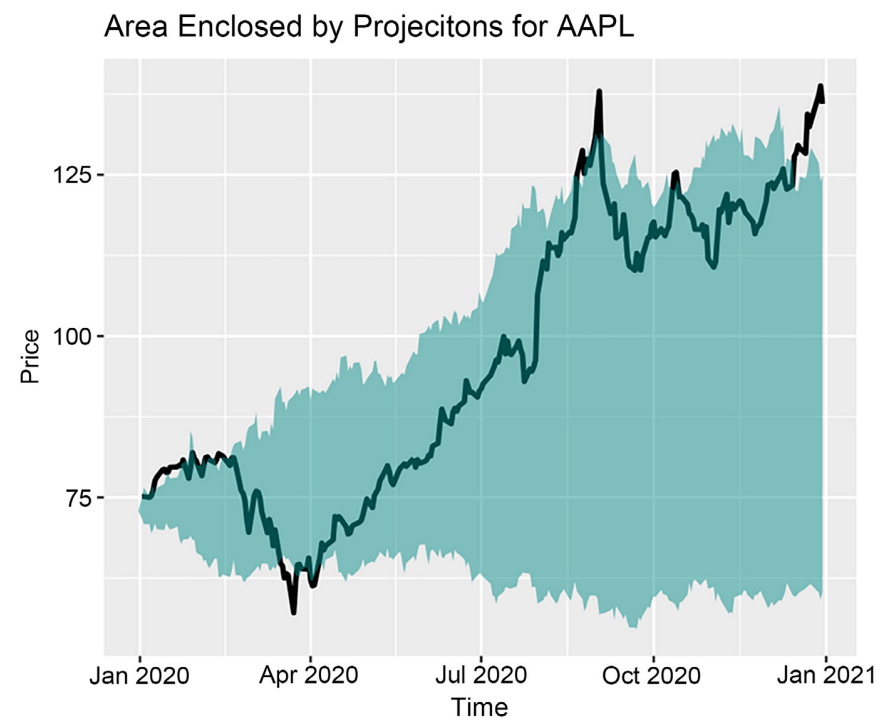

Figure 2. Plot of historical Apple stock price from January 2020 to January 2021 (black) and the area enclosed between the maximal and minimal prices in the simulated paths in figure 1, assuming the law in equation 2 with $\Gamma=0.0008316271$ and $\Sigma=0.01648899$. 


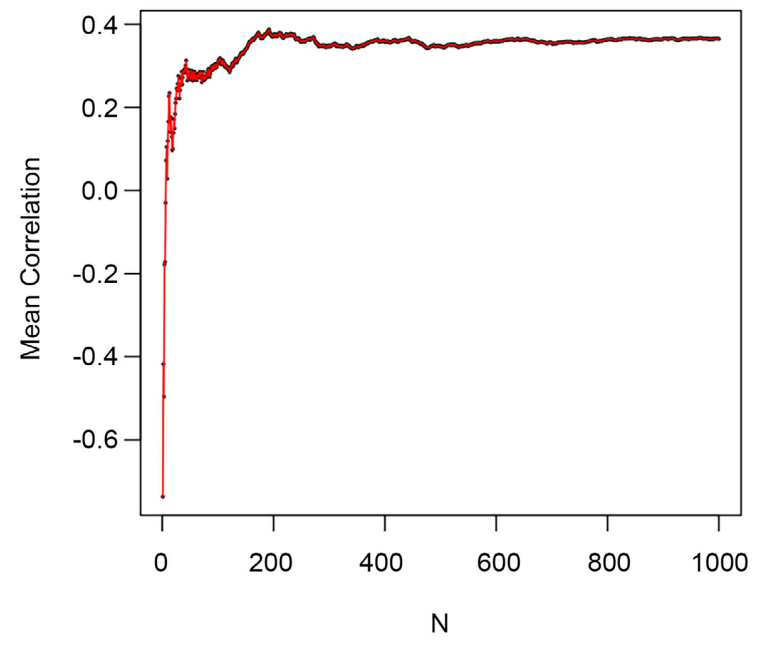

Figure 3. Plot of mean correlation $\operatorname{Cor}^{N}$ for values of $\mathrm{N}$ in $\{1,2,3, \cdots, 1000\}$. The plot seems to converge to a value of approximately 0.36 .

According to basic probability theory, $\mathfrak{C o r}^{N}$ converge in the sense of probability to the expected value of the correlation between the discretised path and the historical trial data $\mathbb{E}\left[\operatorname{Cor}\left(\left.\mathcal{Y}_{t}\right|_{\mathfrak{I}}, H_{t}\right)\right]$ [9]. This means that considering progressively larger values of $N$ (that is taking $N \rightarrow \infty$ ), $\operatorname{Cor}^{N}$ should approach a constant value (see Figure 3).

Figure 3 suggests that

$$
\mathbb{E}\left[\operatorname{Cor}\left(\left.\mathcal{Y}_{t}\right|_{T_{T}}, H_{t}\right)\right] \approx \operatorname{Cor}^{N}
$$

tends to a constant value of 0.36 for $N$ large. This suggests, in a rather heuristic way, that the model "captures" $36 \%$ of the variability in stock prices. Granted, further investigation is warranted over different stocks and more involved models could of course be considered.

\section{Conclusions}

A unique contribution of this report was the empirical verification of a theoretical model for Apple's stock price predicated on Brownian Motion as a quantification of the volatility therein. This was achieved by comparing the projected stock price against historical data from January 12020 to December 312020. The model used data going back to 2007, and used the discretised version of the price process of the Apple Stock. A limiting value of 0.36 was observed for the mean correlation between the theoretical and actual stock prices, upon numerical simulation for a larger and larger sample size. This suggests that the model "captures" $36 \%$ of the variability in the stock price. Granted, the above method has limitations such as the fact that volatility is estimated from historical data. Further investigation is warranted to compute such correlation coefficients over different stocks and one could construct more involved models, could of course be considered (see [10]). 
Since the model considers a deterministic volatility factor, it would be an interesting extension to derive and test the accuracy of a similar model with volatility being purely stochastic. Brownian Motion has seen extensive use in the world of financial mathematics, especially in the construction of pricing models for more involved financial instruments including options and other derivatives (see [11] and [12]).

\section{Conflicts of Interest}

The authors declare no conflicts of interest regarding the publication of this paper.

\section{References}

[1] Brown, R., et al. (1828) XXVII. A Brief Account of Microscopical Observations Made in the Months of June, July and August 1827, on the Particles Contained in the Pollen of Plants; and on the General Existence of Active Molecules in Organic and Inorganic Bodies. The Philosophical Magazine, 4, 161-173. https://doi.org/10.1080/14786442808674769

[2] Bachelier, L. (1900) Théorie de la Spéculation. Annales Scientifiques de l'Ecole Normale Supérieure, 17, 21-86. https://doi.org/10.24033/asens.476

[3] Wiener, N. (1923) Differential-Space. Journal of Mathematics and Physics, 2, 131-174. https://doi.org/10.1002/sapm192321131

[4] Kolmogorov, A.K. and Bharucha-Reid, A.T. (2018) Foundations of the Theory of Probability. 2nd Edition, Courier Dover Publications, New York.

[5] Huo, H. (2006) G Introduction to Stochastic Integration. Springer-Verlag, Berlin.

[6] Øksendal, B. (2003) Stochastic Differential Equations. Springer, New York. https://doi.org/10.1007/978-3-642-14394-6 5

[7] Fernholz, E.R. (2002) Stochastic Portfolio Theory. Springer, Princeton. https://doi.org/10.1007/978-1-4757-3699-1 1

[8] Bremaud, P. (2020) Probability Theory and Stochastic Processes. Springer Nature, Berlin.

[9] Blitzstein, J.K. and Hwang, J. (2019) Introduction to Probability. Chapman and Hall/ CRC, Boca Raton. https://doi.org/10.1201/9780429428357

[10] Heston, S.L. (1993) A Closed-Form Solution for Options with Stochastic Volatility with Applications to Bond and Currency Options. The Review of Financial Studies, 6, 327-343. https://doi.org/10.1093/rfs/6.2.327

[11] Black, F. and Scholes, M. (1973) The Pricing of Options and Corporate Liabilities. Journal of Political Economy, 81, 637-654. https://doi.org/10.1086/260062

[12] Moalosi-Court, K.I. (2019) Pricing European Option When the Stock Price Process Is Being Driven by Geometric Brownian Motion. Open Access Library Journal, 6, e5568. https://doi.org/10.4236/oalib.1105568 